\title{
COMPACTIFICATIONS WITH COUNTABLE REMAINDER
}

\author{
M. G. CHARALAMBOUS
}

\begin{abstract}
In this paper, we deal with the problem of characterizing those spaces that have a compactification with countable remainder.
\end{abstract}

1. Introduction and definitions. A collection $Q$ of subsets of a topological space $X$ is called a network if every open subset of $X$ is the union of a subcollection of $Q . R(X)$ denotes the set of all points of $X$ which possess no compact neighbourhood. If $Y$ is a Hausdorff compactification of $X$, it is readily seen that $R(X)$ is the intersection of $X$ with the closure of $Y-X$ in $Y$. A Hausdorff compactification $Y$ of $X$ is said to have countable remainder if $Y-X$ is a countable set; by an abuse of terminology, we shall say that such a $Y$ is a countable compactification of $X$. In what follows, the space $X$ is assumed to be at least Tychonoff. Two necessary conditions for $X$ to have a countable compactification are (a) $X$ is Čech-complete and (b) $X$ is rim-compact. These are, in fact, sufficient conditions as well in the case when $X$ is metric separable [6], [10]. However, the product of the space of irrational numbers with an uncountable discrete space, despite satisfying both (a) and (b), possesses no countable compactification [4]. There has recently been interest in finding conditions which, together with (a) and (b), ensure that $X$ has a countable compactification ([2], [3], [4], [8]). Terada has shown that one such condition is that $R(X)$ is compact metric, and Hoshina has weakened this to the requirement that $R(X)$ is metric separable. In this paper, we show that (a) and (b), together with the condition that $R(X)$ has a countable network, ensure that $X$ has a countable compactificaion. This includes Hoshina's result as well as the case when $R(X)$ is countable. In addition, our proof is considerably shorter than the one given by Hoshina. Furthermore, we construct examples to show that, in general, the topological properties of $R(X)$ do not determine whether $X$ has a countable compactification.

\section{A result.}

THEOREM. Let $X$ be a Čech-complete, rim-compact space such that $R(X)$ has a countable network. Then $X$ has a countable compactification.

Proof. Since $X$ is rim-compact, $X$ has at least one compactification $Z$ with $\operatorname{ind}(Z-X) \leqslant 0$, where ind denotes small inductive dimension, and since $X$

Received by the editors April 14, 1978 and, in revised form, January 17, 1979.

AMS (MOS) subject classifications (1970). Primary 54D35, 54D40, 54 G20.

Key words and phrases. Rim-compact, Cech-complete, network, countable compactification, small inductive dimension, covering dimension. 
is Čech-complete, $Z-X=\cup_{n=1}^{\infty} F_{n}$, where for each $n$ in $N$, the set of positive integers, $F_{n}$ is compact [5]. Let $\left\{A_{n}: n \in N\right\}$ be a network for $R(X)$. For a fixed $n$ in $N$, let $M=\left\{m \in N: \bar{A}_{m} \cap F_{n}=\varnothing\right\}$. If $x$ is a point of $R(X)$, by regularity of $Z$, there is an open set $V$ of $Z$ and some $m$ in $M$ with $x \in A_{m} \subset V \subset \bar{V} \subset Z-F_{n}$. For each $m$ in $M$, by normality of $Z$, there is a cozero set $G_{m}$ of $Z$ with $A_{m} \subset G_{m} \subset Z-F_{n}$. Put

$$
E_{n}=Z-\bigcup_{m \in M} G_{m} \cup(X-R(X))
$$

It is readily seen that $E_{n}$ is a compact subset of $Z-X$ such that $F_{n} \subset E_{n}$, $Z-X=\cup_{n=1}^{\infty} E_{n}$ and the complement of $E_{n}$ in any compact subset of $\overline{Z-X}=(Z-X) \cup R(X)$ is $\sigma$-compact. We may further assume that $E_{n} \subset$ $E_{n+1}$ for each $n$ in $N$. Now $E_{n+1}-E_{n}$ is a locally compact, $\sigma$-compact space with ind $\left(E_{n+1}-E_{n}\right) \leqslant 0$. Hence $E_{n+1}-E_{n}$ is the union of a countable collection of mutually disjoint compact sets. It follows that $Z-X=$ $\cup_{n=1}^{\infty} B_{n}$, where, for $n, m$ in $N$ with $n \neq m, B_{n}, B_{m}$ are disjoint compact sets, and $\left(Z-X \cup B_{n}\right) \cup R(X)=\cup_{m=1}^{\infty} C_{n, m}$, where $C_{n, m}$ is compact for all $n, m$ in $N$.

Since $Z-X$ is Lindelöf and $\operatorname{ind}(Z-X) \leqslant 0$, then $\operatorname{dim}(Z-X) \leqslant 0$, where $\operatorname{dim}$ denotes covering dimension. Hence, if $E, F$ are disjoint closed sets of $Z$, there exist disjoint open sets $G, H$ with $E \subset G, F \subset H$ and $Z-X \subset$ $G \cup H$ (see e.g. [1, Proposition 4]). It follows that there are pairs $G_{i}, H_{i}$ of disjoint open sets of $Z$ with $(Z-X) \subset G_{i} \cup H_{i}, i \in N$, and such that $E \subset G_{i}$ and $F \subset H_{i}$ for some $i$ in $N$ in each of the following cases. Firstly when $E=B_{n}$ and $F=C_{n, m}$, secondly when $E=\bar{A}_{n}, F=\bar{A}_{m}$ and $\overline{A_{n}} \cap \overline{A_{m}}$ $=\varnothing$, and thirdly when $E=\overline{A_{n}}, \quad F=B_{m}$ and $\overline{A_{n}} \cap B_{m}=\varnothing$, where $n, m$ are in $N$.

We now define an equivalence relation $\sim$ on $Z$ as follows. If $x, y \in B_{n}$ for some $n$ in $N$, then $x \sim y$ if and only if $x$ and $y$ belong to the same member of $\left\{G_{i}, H_{i}\right\}$ for each $i \leqslant n$. Otherwise, $x \sim y$ if and only if $x=y$. Let $\pi: Z \rightarrow Y$ be the quotient map induced by $\sim$. The equivalence class $\pi^{-1} \pi(x)$ of a point $x$ of $B_{n}$ is the closed set $D_{1} \cap \cdots \cap D_{n} \cap B_{n}$, where, for $i<n, D_{i}$ is the member of $\left\{G_{i}, H_{i}\right\}$ which contains $x$. Hence $\pi\left(B_{n}\right)$ consists of a finite number of points. Clearly, $Y$ is a $T_{1}$ compactification of $X$ with $Y-X$ countable. To complete the proof, it suffices to show that $\pi$ is a closed map, since this implies that $Y$ is normal and therefore Hausdorff.

Let $S$ be a closed set of $Z$. Then $\pi^{-1} \pi(S)=S \cup T$, where $T=\cup{ }_{n=1}^{\infty} T_{n}$ and $T_{n}=\pi^{-1} \pi\left(S \cap B_{n}\right)-S$. Let $x$ be a limit point of $T$. It suffices to show that $x \in S \cup T$, since this implies that $\pi^{-1} \pi(S)$ is closed and hence $\pi$ is closed. Since $T$ is a subset of the closed set $(Z-X) \cup R(X)$, either $x \in$ $R(X)$ or, for some $n$ in $N, x \in B_{n}$. We note that, for $m, k$ in $N$, since $\pi\left(B_{m}\right)$ is finite, then $\pi^{-1} \pi\left(S \cap B_{m}\right)$ is closed, so that if $x$ is not in $\cup_{m<k} \pi^{-1} \pi(S \cap$ $\left.B_{m}\right)$, then $x$ is a limit point of $\cup_{m>k} T_{m}$.

We first assume that $x \in R(X)$. Let $K=\left\{k \in N: x \in G_{k} \cup H_{k}\right\}$. For $k$ in 
$K$, write $D_{k}$ for the element of $\left\{G_{k}, H_{k}\right\}$ which contains $x$. Now $x$ is a limit point of $\cup_{m>k} T_{m}$ and hence there is an element $x_{k}$ of this set which is contained in $\cap\left(D_{i}: i \in K, i \leqslant k\right)$. Let $y_{k}$ be an element of $S$ with $y_{k} \sim x_{k}$. Then, for $i<k, y_{k} \in H_{i}$ implies $x_{k} \in H_{i}$. The infinite subset $\left\{y_{1}, y_{2}, \ldots\right\}$ of the compact set $S$ has a limit point $y$ in $S$. Suppose $y \neq x$. Either $y \in R(X)$ or $y \in B_{n}$ for some $n$ in $N$. In the first case, there are open neighbourhoods $U, V$ of $x, y$ with $\bar{U} \cap \bar{V}=\varnothing$ and $m, n$ in $N$ with $x \in A_{m} \subset U$ and $y \in A_{n}$ $\subset V$. Clearly $\overline{A_{m}} \cap \overline{A_{n}}=\varnothing$ and hence there is $r$ in $N$ with $\overline{A_{m}} \subset G_{r}$ and $\bar{A}_{n} \subset H_{r}$. In the second case, let $U$ be a neighbourhood of $x$ with $\bar{U} \cap B_{n}=$ $\varnothing$ and let $m$ be in $N$ with $x \in A_{m} \subset U$. Since $\overline{A_{m}} \cap B_{n}=\varnothing$, there is an $r$ in $N$ with $\bar{A}_{m} \subset G_{r}$ and $B_{n} \subset H_{r}$. Now since $y$ is a limit point of $\left\{y_{1}, y_{2}, \ldots\right\}$, for some $k>r, y_{k} \in H_{r}$, which implies that $x_{k} \in H_{r}$, so that, since $G_{r} \cap H_{r}$ $=\varnothing, x_{k} \notin G_{r}=D_{r}$. This contradicts the fact that $x_{k}$ is in $\cap\left(D_{i}: i \in K\right.$, $i<k$ ) and shows that $x=y$ and hence $x \in S$.

Finally, suppose $x \in B_{n}$ for some $n \in N$. It remains to show that $x \in$ $\pi^{-1} \pi\left(S \cap B_{n}\right)$. Suppose this is false. For $i \in N$, let $D_{i}$ be the member of $\left\{G_{i}, H_{i}\right\}$ which contains $x$. Then $\pi^{-1} \pi(x)=D_{1} \cap \ldots \cap D_{n} \cap B_{n}$ and $S \cap$ $D_{1} \cap \ldots \cap D_{n} \cap B_{n}=\varnothing$. The closure $Q$ of $(S-X) \cap D_{1} \cap \ldots \cap D_{n}$ is a compact subset of $(Z-X) \cup R(X)$ which is disjoint from $B_{n}$. For if $y \in B_{n}$ $\cap Q$, then $y \in B_{n} \cap S$, so that for some $j<n, y \notin D_{j}$, and if $P_{j}$ is the member of $\left\{G_{j}, H_{j}\right\}$ which contains $y$, then $P_{j} \cap Q=\varnothing$. Thus $Q$ is a compact subspace of $\cup_{k=1}^{\infty} C_{n, k}$. Hence there is a finite subset $L$ of $N$ such that $B_{n} \subset G_{i}$ for each $i \in L$ and $Q \subset \cup\left(H_{i}: i \in L\right)$. Let $k=n+\max L$ and $D=D_{1} \cap \ldots \cap D_{k}$. Since $x \in B_{n}$, for $i \in L, D_{i}=G_{i}$. Let $m>k$ and suppose $y \in D \cap T_{m}$. Then there is $z$ in $S \cap B_{m}$ with $y \sim z$. For $i \leqslant k, y$ and $z$ belong to the same element of $\left\{G_{i}, H_{i}\right\}$. Hence $z \in D$ and it follows that $z \in Q$. Therefore for some $i$ in $L, z \in H_{i}$, which is absurd since $G_{i} \cap H_{i}=\varnothing$ and $z \in D \subset D_{i}=G_{i}$. This shows that $x$ is not a limit point of $\cup_{m>k} T_{m}$ and since our assumption that $x \in B_{n}$ and $x \notin \pi^{-1} \pi\left(S \cap B_{n}\right)$ implies that $x$ is not in $\cup_{m<k} \pi^{-1} \pi\left(S \cap B_{m}\right)$, then $x$ is not a limit point of $T$. This contradiction shows that $x$ must be in $\pi^{-1} \pi\left(S \cap B_{n}\right)$ and completes the proof of the theorem.

3. Some examples. Example 1 shows that there are rim-compact, Čech-complete spaces $X, X_{1}$, such that, despite $R(X), R\left(X_{1}\right)$ being homeomorphic, $X$ has a countable compactification but not $X_{1}$. In this example, $R(X)$ is compact. In Example 2, the same pathology is exhibited with $R(X)$ discrete. Hoshina [4] has shown that if a paracompact space $X$ has a countable compactification, then $R(X)$ is Lindelöf. Example 2 shows that, in general, the fact that $X$ has a countable compactification does not imply that $R(X)$ is Lindelöf.

We need the following result of Hoshina [4].

LEMMA. If $X$ has a countable compactification and $\mathcal{Q}$ is a collection of mutually disjoint open sets of $X$ with $U \cap R(X) \neq \varnothing$ for each $U$ in $थ$, then $\mathscr{Q}$ is countable. 
EXAMPLE 1. Let $R$ be the set of real numbers with the usual topology. Then $X=\beta R-N$, where $\beta$ denotes Stone-Čech compactification, has a countable compactification and $R(X)=\beta N-N$ [8, Example 3].

Let $N \cup\{\infty\}$ be the one-point compactification of $N, Y=(N \cup\{\infty\}) \times$ $(N \cup\{\infty\}) \times R(X)$ and $X_{1}=Y-\{\infty\} \times N \times R(X)$. Since $Y$ is compact and $Y-X_{1}$ is $\sigma$-compact and zero-dimensional, then $X_{1}$ is Cech-complete and rim-compact. In addition, $R\left(X_{1}\right)=\{\infty\} \times\{\infty\} \times R(X)$ is homeomorphic with $R(X)$. Let $\mathcal{Q}$ be an uncountable collection of mutually disjoint nonempty open sets of $\beta N-N\left[9\right.$, p. 77]. For each $U$ in $थ$, let $U^{*}=(N \cup$ $\{\infty\}) \times(N \cup\{\infty\}) \times U$. Then $\left\{U^{*} \cap X_{1}: U \in \mathcal{U}\right\}$ is an uncountable collection of mutually disjoint open sets of $X_{1}$ with $U^{*} \cap X_{1} \cap R\left(X_{1}\right) \neq \varnothing$ for each $U$ in $\mathcal{Q}$. The lemma implies that $X_{1}$ has no countable compactification.

EXAMPLE 2. Let $P$ be the set of irrational numbers and $Q$ the set rational numbers. For each $x$ in $P$, let $\left\{x_{1}, x_{2}, \ldots\right\}$ be a sequence of rationals converging to $x$ in the usual topology of $R$. A subset $A$ of $R$ is defined to be open if whenever $x \in A \cap P$, then there is $n$ in $N$ with $\left\{x_{n}, x_{n+1}, \ldots\right\} \subset A$. With this topology, $R$ is locally compact and Hausdorff, $Q$ is dense in $R$ and $P$ is a closed subspace of $R$ with discrete topology [7, p. 87]. Let $R \cup\{\infty\}$ be the one-point compactification of $R, Y=(N \cup\{\infty\}) \times(R \cup\{\infty\})$ and $X=Y-\{\infty\} \times Q \cup\{\infty\}$. Then $Y$ is a countable compactification of $X$, while $R(X)=\{\infty\} \times P$ is not Lindelöf.

Let $Z=(N \cup\{\infty\}) \times Y$ and $X_{1}=(Z-\{\infty\} \times Y) \cup\{\infty\} \times\{\infty\} \times P$. Then $X_{1}$ is Čech-complete and rim-compact, because $Z-X_{1}$ is $\sigma$-compact and zero-dimensional, and $R\left(X_{1}\right)=\{\infty\} \times\{\infty\} \times P$ is homeomorphic with $R(X)$. However, the lemma implies that the closed subspace $N \times(N \cup\{\infty\})$ $\times(P \cup\{\infty\}) \cup R\left(X_{1}\right)$ of $X_{1}$ has no countable compactification, and hence $X_{1}$ has no countable compactification.

We can obviously choose $X, X_{1}$ so that $R(X), R\left(X_{1}\right)$ are homeomorphic with the one-point compactification of $P$.

\section{BIBLIOGRAPHY}

1. M. G. Charalambous, Spaces with increment of dimension n, Fund. Math. 93 (1976), 97-107.

2. M. Henriksen, Tychonoff spaces that have a compactification with a countable remainder, General Topology and its Relations to Modern Analysis and Algebra, IV (Proc. Fourth Prague Topological Sympos., Prague, 1976), Part B, Soc. Czech. Mathematicians and Physicists, Prague, 1977, pp. 164-167.

3. T. Hoshina, Compactifications by adding a countable number of points, General Topology and its Relations to Modern Analysis and Algebra, IV, Part B, Soc. Czech. Mathematicians and Physicists, Prague, 1977, pp. 168-169.

4. Countable-points compactifications for metric spaces, preprint.

5. J. R. Isbell, Uniform spaces, Math. Surveys, no. 12, Amer. Math. Soc., Providence, R. I., 1964.

6. K. Morita, On bicompactifications of semibicompact spaces, Sci. Rep. Tokyo Bunrika Daigaku, Sect. A 4 (1952), 222-229.

7. L. A. Steen and J. A. Seebach, Jr., Counterexamples in topology, Holt, Rinehart and Winston, New York, 1970.

8. T. Terada, On countable discrete compactifications, General Topology and Appl. 7 (1977), 321-327. 
9. R. C. Walker, The Stone- $\check{C} e c h$ compactification, Ergebnisse der Mathematik und ihrer Grenzgebiete, Band 83, Springer-Verlag, New York and Berlin, 1974.

10. L. Zippin, On semicompact spaces, Amer. J. Math. 57 (1935), 327-341.

Department of Mathematics, Ahmadu Bello University, Zaria, Nigeria

Current address: Department of Mathematics, University of Nairobi, P. O. Box 30917, Nairobi, Kenya 